\title{
Entwicklung einer kraftausdauerorientierten Testbatterie als Entscheidungshilfe für eine leistungsorientierte Gruppenzuwei- sung in der rehabilitativen Sporttherapie bei muskuloskeletalen Krankheiten
}

\author{
Development of a Strength-Endurance Oriented Assessment as \\ Decision Aid for a Performance Oriented Group Assignment to \\ Rehabilitative Exercise Therapy of Patients with Musculoskeletal \\ Diseases
}

Autoren

André Golla ${ }^{1}$, Kerstin Mattukat ${ }^{1}$, Inge Ehlebracht-König² ${ }^{2}$ Karin Kluge ${ }^{3}$, Klaus Pfeifer ${ }^{4}$, Wilfried Mau ${ }^{1}$

Institute

1 Institut für Rehabilitationsmedizin, Medizinische Fakultät der Martin-Luther-Universität Halle-Wittenberg, Halle (Saale)

2 Rheuma-Liga Niedersachsen e.V., Hannover

3 CELENUS Teufelsbad Fachklinik, Blankenburg

4 Institut für Sportwissenschaft und Sport, Friedrich-Alexander-Universität Erlangen-Nürnberg, Erlangen

\section{Schlüsselwörter}

Sporttherapie, Leistungsorientierung, motorischer Test, medizinische Rehabilitation, muskuloskeletale Krankheiten

Key words

exercise therapy, performance orientation, motoric test, medical rehabilitation, musculoskeletal diseases

eingereicht 08.11 .2016

akzeptiert 22.02.2017

Bibliografie

DOI https://doi.org/10.1055/s-0043-104533

Phys Med Rehab Kuror 2017; 27: 149-159

(c) Georg Thieme Verlag KG Stuttgart · New York ISSN 0940-6689

Korrespondenzadresse

André Golla

Institut für Rehabilitationsmedizin

Martin-Luther-Universität Halle-Wittenberg

Magdeburger Straße 8, 06112 Halle (Saale)

andre.golla@medizin.uni-halle.de

\section{ZUSAMMENFASSUNG}

Hintergrund Vor dem Hintergrund des breiten Leistungsniveaus von Patienten in der medizinischen Rehabilitation und dem trainingswissenschaftlichen Anspruch einer optimalen Belastungsdosierung fehlt es bislang an praxistauglichen Entscheidungshilfen für eine leistungsorientierte Ausgestaltung von sport- und bewegungstherapeutischen Gruppenangeboten.

Methoden Daher wurde eine kraftausdauerorientierte, diagnoseübergreifende Testbatterie für die Unterteilung von Rehabilitanden in 2 unterschiedliche Leistungsgruppen entwickelt (moderat vs. intensiv belastbar). An einer Stichprobe von 163 Rehabilitanden mit verschiedenen muskuloskeletalen Krankheiten (Rückenschmerzen, chronischen Polyarthritiden bzw. Spondyloarthritiden) wurden die Zuteilungsergebnisse der Testbatterie mit dem Standardverfahren, d. h. einer Unterteilung durch ärztliche Einschätzung, anhand von funktionsbezogenen Parametern verglichen.

Ergebnisse Jeder dritte Rehabilitand wurde durch die beiden Beurteilungsvarianten (Arzt vs. Testbatterie) unterschiedlich bewertet. Bei der testbasierten Unterteilung traten deutlich größere Unterschiede zwischen den moderat und intensiv belastbaren Rehabilitanden bzgl. der gemessenen und der fragebogenbasierten Funktionsparameter auf als bei der Unterteilung auf Basis der ärztlichen Begutachtung.

Schlussfolgerung Die durchgeführte Analyse liefert Hinweise auf eine stärkere Differenzierung des trainingsbezogenen Leistungsvermögens durch eine testbasierte Einschätzung im Vergleich zu einer ärztlichen Beurteilung im Rahmen der Anamnese und Aufnahmeuntersuchung. Für spezifische Therapieentscheidungen könnten die Ergebnisse solcher Entscheidungshilfen wertvolle Informationen liefern. Insbesondere für die klinikinterne Entwicklung von sport- und bewegungstherapeutischen Entscheidungshilfen kann die beschriebene Testentwicklung eine Orientierung darstellen. 


\section{ABSTRACT}

Background For the design of a performance-oriented group therapy in medical rehabilitation, we took 2 aspects into consideration: the wide range of patient's physical capacity and, on the other hand, the demand for an optimal training load for each patient. In daily practice, there is a lack of useful decision aids for a performanceoriented group allocation.

Methods We developed a strength-endurance oriented assessment to classify patients into 2 groups with different physical performance (moderate vs. intensive). To evaluate an additional benefit in terms of patient's physical capacity, we compared the assessment-based results with the results of a medical rating (standard method) in a sample of 163 patients with different musculoskeletal diseases (chronic back pain, polyarthritis or ankylosing spondylitis).

Results The 2 procedures (physician vs. assessment) classified every third patient differently. Regarding function-related parameters, the assessment-based classification demonstrated significantly higher differences between moderate and intensive group compared to the results of of the physicians' rating.

Conclusions The newly developed assessment leads to an improved performance-oriented classification of patients into 2 performance groups compared to a physician's rating which is primarily based on a medical examination. The described approach is an example for the development of practical decision aids in everyday clinical practice.

\section{Einleitung}

In der medizinischen Rehabilitation gehören sport- und bewegungstherapeutische Angebote zum umfangstärksten Leistungssegment [1,2]. Die quantitative Bedeutung steht dabei in Diskrepanz zu der bisher eher geringen Konzeptualisierung dieses Therapiebereiches. So wird durch die Arbeitsgruppe Bewegungstherapie der Deutschen Gesellschaft für Rehabilitationswissenschaften die bewegungstherapeutische Praxis im rehabilitativen Setting als vornehmlich erfahrungsgeleitet und wenig strukturiert bewertet [3]. Vor diesem Hintergrund wurden innerhalb der letzten Jahre wichtige Impulse für eine evidenzbasierte Weiterentwicklung der Bewegungstherapie gesetzt [3-7] und verschiedene Forschungsprojekte initiiert [8-13].

Zu den von der Arbeitsgruppe geforderten, aber bisher kaum bearbeiteten Punkten gehört die „Entwicklung von praktisch nutzbaren Assessmentverfahren als Basis für die Entwicklung spezifischer bewegungstherapeutischer Therapiepfade bei Rehabilitanden mit unterschiedlichen Einschränkungen der funktionalen Gesundheit“ [3]. Für eine patientenorientierte Ausgestaltung der Bewegungstherapie (u. a. nach Art, Inhalt und Dosis) gilt die Berücksichtigung des individuellen Leistungsniveaus der Rehabilitanden ${ }^{1}$ als essentiell [14]. Gerade für sporttherapeutische Interventionen, die nahezu ausschließlich im Gruppensetting realisiert werden [1], stellt die Vermeidung von physischer Über- oder Unterforderung eine besondere Herausforderung dar. Obwohl trainingsmethodische Optionen für das Gruppentraining zur Verfügung stehen (z. B. Fahrtspiel im Ausdauerbereich, Zirkeltraining im Kraftbereich), gefährdet ein ausgeprägtes Leistungsgefälle das Setzen eines optimalen Trainingsreizes für den Einzelnen.

Mithilfe einer leistungsorientierten Diagnostik, wie sie in anderen Bereichen der medizinischen Rehabilitation zu finden ist (z. B. rehabilitationsrelevante Diagnostik bei Herzinsuffizienz [15], berufsbezogene Diagnostik in der MBOR $^{2}$ [16]), könnte die körperliche Belastbarkeit der Rehabilitanden mit muskuloskeletalen Krankheiten zu Rehabilitationsbeginn objektiviert, Kontraindikatoren für verschiedene Therapieangebote und Aktivitätsformen frühzeitig

\footnotetext{
${ }^{1}$ Im Interesse einer besseren Lesbarkeit wird nicht ausdrücklich in geschlechtsspezifischen Personenbezeichnungen differenziert. Die gewählte männliche Form schließt eine adäquate weibliche Form gleichberechtigt ein 2 MBOR = medizinisch-beruflich orientierte Rehabilitation
}

identifiziert und bewegungstherapeutische Gruppenangebote, insbesondere in größeren Kliniken, effizienter geplant und gesteuert werden. Bislang fehlt es allerdings an geeigneten, ökonomischen Entscheidungshilfen für eine leistungsorientierte Gruppenausgestaltung in der rehabilitativen Sport- und Bewegungstherapie. Zwar gibt es eine Vielzahl an motorischen Funktionstests [17] sowie das Konzept der motorischen Basisdiagnostik speziell für den rehabilitativen Bereich [14], jedoch ist deren Praxistauglichkeit aufgrund der organisatorischen Rahmenbedingungen eingeschränkt. Spezifische Funktionstests beziehen sich meist nur auf einzelne Komponenten der Leistungsfähigkeit, zudem fehlen in der Regel geeignete Interpretations- und Vergleichsmöglichkeiten für die rehabilitative Praxis. Bei der heterogenen Testbatterie der motorischen Basisdiagnostik liegt der primäre Fokus zunächst auf der Identifizierung von motorischen Auffälligkeiten (z. B. Rumpfaufrichten möglich/nicht möglich), über die sich stufenweise erst ein weiterer Diagnostikbedarf in speziellen Problembereichen ableiten lässt [18]. Die z.T. geringen Anforderungen der motorischen Basisdiagnostik lassen jedoch keine quantitative Beurteilung der Belastbarkeit zu, wodurch die Möglichkeit zur leistungsorientierten Gruppenbildung kaum gegeben ist.

Sport- und bewegungstherapeutische Gruppenangebote bewegen sich daher häufig im Spannungsfeld zwischen variabler Belastbarkeit der Rehabilitanden, dem trainingswissenschaftlichen Anspruch einer optimalen Belastungsdosierung und den therapeutischen Möglichkeiten in der Praxis. Vor diesem Hintergrund wurde als studienbegleitendes Teilprojekt in der boRN³-Studie [19] eine gruppentaugliche Testbatterie für Rehabilitanden mit verschiedenen muskuloskeletalen Krankheiten entwickelt, deren methodischer Entwicklungsprozess im vorliegenden Beitrag detailliert beschrieben wird. Bewusst wurden verschiedene entzündliche und nicht-entzündliche muskuloskeletale Krankheiten eingeschlossen, weil die Diagnose keine ausreichenden Informationen hinsichtlich der Funktionsfähigkeit bietet. Das wesentliche Ziel bestand in der Generierung einer funktionsorientierten Testbatterie, die sich diagnoseunabhängig anwenden lässt und Rehabilitanden entsprechend ihrer individuellen körperlichen Belastbarkeit und nicht nach bzw. nur innerhalb der rehabilitationsrelevanten Hauptdiagnose sondiert. Mittels die-

\footnotetext{
${ }^{3}$ boRN $=$ „Gestufte bewegungsorientierte Rehabilitation und Nachsorge bei Patienten mit entzündlichen und nicht-entzündlichen Erkrankungen des Bewegungssystems“ (FKZ: 0421-FSCP-Z237)
} 
ser standardisierten Entscheidungshilfe sollten Rehabilitanden mit 3 unterschiedlichen Hauptdiagnosen entweder körperlich moderatem oder intensivem diagnoseunabhängigen Gruppentraining zugeordnet werden. Dies soll gemäß der Internationalen Klassifikation der Funktionsfähigkeit, Behinderung und Gesundheit geforderten funktionsorientierten Ausrichtung der medizinischen Rehabilitation Rechnung tragen und gleichzeitig den Rehabilitationskliniken eine effiziente Möglichkeit zur standardisierten, diagnoseübergreifenden Gruppenzusammenstellung ermöglichen.

Im empirischen Teil dieses Beitrags wird die Gruppenzuordnung durch die Testbatterie mit der Gruppeneinteilung basierend auf der ärztlichen Aufnahmeuntersuchung verglichen, um Hinweise für den potentiellen Mehrwert eines solchen motorischen Assessments zu generieren. Es wird somit ein Verfahren zur klinikinternen Entwicklung von sport- und bewegungstherapeutischen Entscheidungshilfen skizziert und dessen möglicher Mehrwert geprüft.

Dadurch soll ein Beitrag zur praxisorientierten Weiterentwicklung von sport- und bewegungstherapeutischen Assessments im rehabilitativen Kontext geleistet werden.

\section{Material und Methoden}

\section{Datenquelle}

Im Rahmen der sequenziell gestalteten boRN-Studie wurde die Testbatterie anhand der Teilnehmer der Kontrollgruppe $(n=414$, Rekrutierungszeitraum 05/2011-11/2011) und anschließend in einer kleineren Pilotierungsgruppe ( $n=33$, Rekrutierungszeitraum 01/2012-02/2012) entwickelt und anschließend in der Interventionsphase ( $n=323$, Rekrutierungszeitraum 03/2012 -10/2012) zur Zuweisung der Studienteilnehmer zu einer von 2 leistungsgestuften Trainingsgruppen eingesetzt. Für alle an der Entwicklung und Erprobung der Testbatterie beteiligten Rehabilitanden galten folgende Einschlusskriterien: (I) männliche und weibliche Rehabilitanden im Alter zwischen 18 und 65 Jahren mit der Hauptdiagnose chronischer Rückenschmerz (RS; ICD-10: M51-M54), chronische Polyarthritiden (CP; ICD-10: M05-M07) oder Spondyloarthritiden
(SpA; ICD-10: M45-M46); (II) keine Operation innerhalb der letzten 3 Monate; (III) für Sport- und Bewegungstherapie in der Gruppe (ärztliche Beurteilung) geeignet; (IV) Rehabilitationszugang: Heilverfahren der Deutschen Rentenversicherung (allgemeines Antragsverfahren; keine Anschlussrehabilitation). Die boRN-Studie (inkl. Testentwicklung) wurde durch die Ethikkommission der Medizinischen Fakultät der Martin-Luther-Universität Halle-Wittenberg positiv begutachtet und im Rehazentrum Bad Eilsen sowie der Celenus Teufelsbad Fachklinik Blankenburg durchgeführt.

\section{Entwicklungsprozess der Testbatterie}

Die Entwicklung der Testbatterie erfolgte gestuft in Anlehnung an die testtheoretischen Grundlagen der motorischen Diagnostik [20]. Im Vordergrund standen die Praxistauglichkeit sowie die Nutzbarkeit der Testbatterie als Instrument der gruppenbasierten Zuweisung der Probanden zu einer von 2 Trainingsgruppen mit unterschiedlichem Belastungsniveau. In einem ersten Schritt wurden relevante Funktionstests mit Sporttherapeuten aus den beiden Kooperationskliniken sondiert und hinsichtlich ihrer Sinnhaftigkeit und Praktikabilität diskutiert. Die Kriterien der Testauswahl bezogen sich auf die Gruppentauglichkeit der Tests, deren krankheitsübergreifende Einsatzmöglichkeiten sowie deren Einfachheit und Nähe zum Klinikalltag. Weiterhin sollten sportmotorische Tests integriert werden, bei denen die Belastbarkeit der Probanden möglichst metrisch erfasst wird. Der Vorteil dieses Vorgehens bestand darin, dass die Belastbarkeit der Rehabilitanden in unterschiedlichen Abstufungen abgebildet werden konnte. Die Schritte zur Auswahl potentiell geeigneter Tests sind in $>$ Tab. 1 dargestellt.

Insgesamt 414 Rehabilitanden der Kontrollgruppe absolvierten diese Testbatterie zu Beginn ihrer medizinischen Rehabilitation (Alter: $\mathrm{M}=48,8$ [ $\pm 8,3$ ] Jahre; $47 \%$ Frauen; Diagnose: 64,7\% RS; 24,2\% cP; 11,1\% SpA). Neben der Dokumentation der Testergebnisse bewerteten die verantwortlichen Testleiter unter Berücksichtigung der gesamten Testergebnisse sowie aufgrund ihrer persönlichen Erfahrung die körperliche Belastbarkeit der einzelnen Rehabilitanden mit der dichotomen Einschätzung „intensiv belastbar“ vs. „moderat belastbar“.

- Tab. 1 Entwicklungsschritte der Testbatterie.

Testinhalte während der Entwicklungsschritte

\begin{tabular}{|c|c|c|c|}
\hline & $\begin{array}{l}\text { Schritt } 1 \text { - Entwicklung } \\
n=414\end{array}$ & $\begin{array}{l}\text { Schritt } 2 \text { - Modifizierung } \\
n=33\end{array}$ & $\begin{array}{l}\text { Schritt } 3 \text { - Erprobung } \\
\mathrm{n}=323\end{array}$ \\
\hline Funktionstests & $\begin{array}{l}\text { 6-Minuten-Gehtest [21] } \\
\text { Kniebeugen (1 min) } \\
\text { Rumpfbeugen ( } 1 \text { min) } \\
\text { Biering-Sörensen-Test (4 min) [22] } \\
\text { Tandemgang }\end{array}$ & $\begin{array}{l}\text { 6-Minuten-Gehtest } \\
\text { Kniebeugen ( } 3 \mathrm{~min}) \\
\text { Rumpfbeugen ( } 3 \mathrm{~min}) \\
\text { Biering-Sörensen-Test ( } 4 \mathrm{~min}) \\
\text { Stern-Test [23] }\end{array}$ & $\begin{array}{l}\text { 6-Minuten-Gehtest } \\
\text { Kniebeugen ( } 3 \mathrm{~min}) \\
\text { Rumpfbeugen ( } 3 \mathrm{~min}) \\
\text { Biering-Sörensen-Test (4 min) }\end{array}$ \\
\hline Therapeuten & $\begin{array}{l}\text { Auffälligkeit Gangbild } \\
\text { Auffälligkeit Testdurchführung }\end{array}$ & $\begin{array}{l}\text { Auffälligkeit Gangbild } \\
\text { Auffälligkeit Testdurchführung }\end{array}$ & $\begin{array}{l}\text { Auffälligkeit Gangbild } \\
\text { Auffälligkeit Testdurchführung }\end{array}$ \\
\hline Rehabilitanden & $\begin{array}{l}\text { Subjektive Beanspruchung je Test } \\
\text { (Borg-Skala [24]) } \\
\text { Druck- und Bewegungsschmerz } \\
\text { Funktionseinschränkungen }\end{array}$ & $\begin{array}{l}\text { Subjektive Beanspruchung je Test } \\
\text { (Borg-Skala) } \\
\text { Druck- und Bewegungsschmerz } \\
\text { Funktionseinschränkungen }\end{array}$ & $\begin{array}{l}\text { Globalurteil zur eigenen } \\
\text { körperlichen Leistungsfähigkeit } \\
\text { (NRS: } 0-10 \text { ) }\end{array}$ \\
\hline
\end{tabular}


- Tab. 2 Ermittelte Cut-Off-Werte für die 4 finalen Tests.

\begin{tabular}{|l|c|c|c|c|c|c|}
\hline Test & Cut-Off & Sensitivität & Spezifität & AUC & 95\%-KI & P \\
\hline 6-Minuten-Gehtest (Meter) & 560 & 0,762 & 0,719 & 0,818 & $0,779-0,857$ & $<0,001$ \\
\hline Kniebeugen (Anzahl) & 30 & 0,917 & 0,684 & 0,794 & $0,633-0,955$ & 0,007 \\
\hline Rumpfbeugen (Anzahl) & 30 & 0,750 & 0,737 & 0,789 & $0,629-0,950$ & 0,007 \\
\hline Biering-Sørensen-Test (Sekunden) & 60 & 0,773 & 0,787 & 0,839 & $0,798-0,880$ & $<0,001$ \\
\hline AUC = Area under the curve; 95\%-KI = 95\%-Konfidenzintervall, $\mathrm{p}=$ Signifikanzniveau & & & & \\
\end{tabular}

Anhand dieser Einschätzung wurde in einem zweiten Schritt mittels binär-logistischer Regressionsanalyse geprüft, wie gut die Belastbarkeit der Probanden (abhängige Variable: Testleiterurteil „moderat belastbar“ = 0 vs. ,intensiv belastbar“ $=1$ ) durch die einzelnen Testergebnisse und zusätzlich erhobene Parameter auf Rehabilitanden- und Therapeutenebene vorhergesagt werden konnte. Aufgrund dieser Ergebnisse wurde die Testbatterie noch einmal leicht angepasst:

- Der niedrigschwellige, gleichgewichtsorientierte Tandemgang wurde aufgrund nahezu fehlender Leistungsvarianz durch den anspruchsvolleren Stern-Test [23] ersetzt.

- Aufgrund ebenfalls sehr deutlicher Deckeneffekte wurde die maximal erreichbare Wiederholungszahl bei den Knie- und Rumpfbeugen von 25 auf 75 erhöht (statt 1 min nun 3 min Zeit; vorgegebene Kadenz: mindestens 25 Wiederholungen/ $\min )$.

Die überarbeitete Testbatterie wurde an der Pilotgruppe mit insgesamt 33 Rehabilitanden in den beiden Studienzentren getestet. Im abschließenden dritten Schritt wurde die Testbatterie aus ökonomischen Überlegungen heraus auf die aussagekräftigsten Tests reduziert. So wurde der Stern-Test aufgrund mangelnder Aussagekraft für die Vorhersage der körperlichen Belastbarkeit und zu hoher Komplexität nicht mehr eingesetzt. Weiterhin wurde die probandenseitige Einschätzung der Anstrengung bei jedem einzelnen Test auf eine globale Selbstbewertung der eigenen körperlichen Leistungsfähigkeit reduziert (Nummerische Rating-Skala 0 = „gar nicht leistungsfähig“ bis 10 = „voll leistungsfähig“). Die detaillierte Dokumentation der Problembereiche der Patienten hinsichtlich Druck- oder Bewegungsschmerz sowie Funktionseinschränkungen der Gelenke und der Wirbelsäule mittels Schmerzmännchen [25] wurde ebenfalls aufgegeben, da sich hier keinerlei Zusammenhänge zur körperlichen Belastbarkeit der Patienten zeigten.

Für die 4 final ausgewählten Funktionstests wurde mittels Receiver-Operating-Characteristic (ROC) Analyse jeweils ein testspezifischer Cut-Off-Wert ermittelt. In die Analysen gingen das dichotome Testleiterurteil als Zustandsvariable und das jeweilige Ergebnis des Funktionstest als Testvariable ein. Der Cut-Off-Wert zur Unterteilung in moderat und intensiv belastbare Rehabilitanden wurde anhand des Verhältnisses von Sensitivität und Spezifität abgeleitet. Dabei galt als optimaler Schwellenwert jeweils der Testwert, bei dem Sensitivität und Spezifität gleichzeitig $\geq 0$,7 betrugen. Bei mehreren möglichen Werte-Kombinationen wurde der Testwert mit der höchsten Sensitivität gewählt. Die berechneten testspezifischen Cut-Off-Werte zur Unterteilung der Rehabilitan- den in moderat und intensiv belastbare Trainingsgruppen sind in - Tab. 2 dargestellt. Bei allen Cut-Off-Werten nahm die,Area under the Curve ( $A \cup C$ ) einen Wert von>0,7 ein und spricht damit für eine akzeptable Modellgüte.

Auswertungsschema der Testbatterie Die finalen Inhalte der Testbatterie sind in \ Tab. $\mathbf{3}$ beschrieben. Es lassen sich 4 dichotome Leistungsindizien ableiten, die zusammen als Entscheidungsgrundlage der Gruppenallokation für die Testleiter dienen. Liegt z. B. die zurückgelegte Strecke beim 6-Minuten-Gehtest über $560 \mathrm{~m}$ wird dies als intensive Leistungsindiz gewertet, ein Testergebnis unter 560 m entspricht einem moderaten Leistungsindiz. Überwiegend intensive Leistungsindizien („।“) gelten als Hinweis auf einen eher intensiv belastbaren Rehabilitanden, während überwiegend moderate Leistungsindizien („M“) eher für einen moderat belastbaren Probanden sprechen. Bei ausgeglichenem Verhältnis (z. B. 2-mal „M“ und 2-mal „।“) können vorhandene Auffälligkeiten bei der Testdurchführung (wenn vorhanden = moderates Leistungsindiz) und die Rückmeldung des Rehabilitanden zur derzeitigen Leistungsfähigkeit herangezogen werden, um dessen individuelle Leistungskapazitäten/-reserven besser abschätzen zu können. Die endgültige Einstufung des Rehabilitanden obliegt dem Testleiter, der z. B. bei Ergebnissen nahe am Cut-Off-Wert oder bei nicht erhobenen Kontraindikatoren eine angemessene Allokationsentscheidung trifft.

Material und Durchführung Für die Testbatterie hat sich während der Entwicklungsphase ein Testleiter-Probanden-Schlüssel von 1:10 als geeignet erwiesen. Im Rahmen der Studie wurden die Tests von Physio- und Sporttherapeuten angeleitet. Die Testbatterie kann inklusive der Erläuterung und Vorbereitung der Tests bei entsprechender Routine innerhalb von 60 min mit der Gruppe durchgeführt werden. Als Materialien werden Begrenzungsmarkierungen für die Gehstrecke, eine Stoppuhr, Gymnastikmatten und Steppbretter benötigt. Für die Vorgabe der Wiederholungsfrequenz empfiehlt sich ein Metronom bzw. ein elektronischer Signalgeber (z. B. Smartphone).

\section{Vorgehen zum Vergleich der Gruppenzuteilung aufgrund der Ergebnisse der Testbatterie und der ärztlichen Einschätzung}

In der boRN-Studie wurden die beteiligten Klinikärzte bezüglich der Intention zur Bildung leistungsgestufter, krankheitsübergreifender, geschlossener Trainingsgruppen während der Interventionsphase sensibilisiert. Sie wurden gebeten, auf Basis ihrer Anamnese jeden Rehabilitanden als „moderat belastbar“ $\left(\mathrm{M}_{\mathrm{A}}\right)$ oder ,intensiv belast- 
- Tab. 3 Übersicht zur Zusammensetzung der finalen Testbatterie.

\begin{tabular}{|c|c|c|}
\hline Komponenten & Kurzbeschreibung & Parameter \\
\hline 6-Minuten-Gehtest & $\begin{array}{l}\text { Eine abgemessene Strecke wird (wiederholt) gehend zurückgelegt. Der } \\
\text { Proband soll in } 6 \mathrm{~min} \text { so weit wie möglich gehen. }\end{array}$ & Gehstrecke in Metern \\
\hline Kniebeuge & $\begin{array}{l}\text { Der Teilnehmer macht möglichst viele Kniebeugen in } 3 \text { min. Die Oberschenkel } \\
\text { befinden sich dabei nahezu parallel zum Boden. Frequenz: mindestens } 25 \\
\text { Wiederholungen/min (Metronom) }\end{array}$ & $\begin{array}{l}\text { Anzahl korrekter Wiederholungen bis } \\
\text { Abbruch (max. } 3 \mathrm{~min} \text { ) }\end{array}$ \\
\hline Rumpfaufrichten & $\begin{array}{l}\text { Der Teilnehmer macht möglichst viele Rumpfbeugen in } 3 \text { min. Frequenz: } \\
\text { mindestens } 25 \text { Wiederholungen/min (Metronom) }\end{array}$ & $\begin{array}{l}\text { Anzahl korrekter Wiederholungen bis } \\
\text { Abbruch (max. } 3 \mathrm{~min} \text { ) }\end{array}$ \\
\hline Biering-Sørensen-Test & $\begin{array}{l}\text { Der Teilnehmer liegt mit der Hüfte auf einem Steppbrett auf und hält den } \\
\text { Oberkörper horizontal zum Boden (Beine fixiert). }\end{array}$ & $\begin{array}{l}\text { Zeit in Sekunden in der Zielposition bis } \\
\text { zum Abbruch (max. } 240 \text { s) }\end{array}$ \\
\hline $\begin{array}{l}\text { Auffälligkeiten im } \\
\text { Gangbild }\end{array}$ & $\begin{array}{l}\text { Beurteilung des Gangbilds beim 6-Minuten-Gehtest hinsichtlich funktions- } \\
\text { bezogener Auffälligkeiten z. B. Trendelenburg-Zeichen, Duchenne-Hinken, } \\
\text { häufige Pausen }\end{array}$ & nicht vorhanden/vorhanden \\
\hline $\begin{array}{l}\text { Probleme bei der } \\
\text { Testdurchführung }\end{array}$ & $\begin{array}{l}\text { Beurteilung von Problemen z. B. beim Hinlegen oder Aufstehen von der Matte } \\
\text { bei den beiden letzten Tests }\end{array}$ & nicht vorhanden/vorhanden \\
\hline $\begin{array}{l}\text { Selbsteinschätzung } \\
\text { der Probanden }\end{array}$ & Wie schätzen Sie Ihre derzeitige körperliche Leistungsfähigkeit ein? & $\begin{array}{l}0=\text { „gar nichtleistungsfähig“ bis } 10=\text { „voll } \\
\text { leistungsfähig“ }\end{array}$ \\
\hline
\end{tabular}

bar“ $\left(I_{A}\right)$ zu bewerten und dies zu dokumentieren. Nach der ärztlichen Anamnese durchliefen alle Rehabilitanden die Testbatterie und wurden über den Zuweisungsalgorithmus ebenfalls als „moderat belastbar“ $\left(M_{T}\right)$ oder „intensiv belastbar“ $\left(I_{T}\right)$ eingestuft. Auf diese Weise konnte die ärztliche Einschätzung unabhängig vom Ergebnis der Testbatterie erhoben und mit diesem verglichen werden.

Zur Identifizierung des möglichen Mehrwerts der testbasierten Gruppenbildung gegenüber der alleinigen ärztlichen Eingruppierung wurden alle Rehabilitanden am Rehabilitationsbeginn zum selbstwahrgenommenem Funktionsstatus schriftlich befragt. Zur Anwendung kamen der Fragebogen zur Erfassung des motorischen Funktionsstatus (FFB-Mot) [26], der Index zur Messung von Einschränkungen der Teilhabe (IMET) [27], ein Fragebogen zur Erfassung der gesundheitsbezogenen Lebensqualität (SF-12) [28] und Skalen zu Schmerzen, Erschöpfung und Morgensteifheit (Numerische Rating-Skalen: 0-10). Alle Fragebogenparameter wurden getrennt nach ärztlicher und testbasierter Belastungseinstufung $\left(\mathrm{M}_{A}\right.$ und $\mathrm{I}_{\mathrm{A}}$ vs. $\mathrm{M}_{\mathrm{T}}$ und $\mathrm{I}_{\mathrm{T}}$ ) zur Abschätzung der Konvergenzvalidität beider Verfahren gegenübergestellt. Die Mittelwertdifferenz zwischen den Gruppen der moderat und intensiv belastbaren Rehabilitanden wurde als Maß einer leistungsorientierten Gruppenzuweisung gewertet. Es kann angenommen werden, dass sich die Gruppen mit größeren Mittelwertunterschieden in den Funktionsparametern hinsichtlich der körperlichen Belastbarkeit deutlicher voneinander unterscheiden (moderate vs. intensive Gruppe). Für die Analyse wurden nur Datensätze der Interventionsgruppe berücksichtigt, bei denen alle benötigten Informationen (alle Fragebogenitems und die ärztliche Belastungseinschätzung) vollständig dokumentiert waren.

\section{Statistik}

Die Ergebnisse wurden für die Gesamtstichprobe sowie die ärztliche und testbasierende Unterteilung in moderate bzw. intensive belastbare Rehabilitanden deskriptiv aufbereitet. Sofern nicht anders angegeben, werden Mittelwert (M) und Standardabweichung (SD) bzw. prozentuale Anteile dargestellt. Lineare Zusammenhänge zwischen den Ergebnissen der 4 final ausgewählten Tests wurden mittels Pearson-Korrelation ( $r$ geprüft. Zur Kontrolle von testbezogenen Leistungsunterschieden zwischen den Diagnosegruppen wurde für jeden Test eine einfaktorielle Varianzanalyse mit Post-Hoc-Tests nach Scheffé durchgeführt. Aufgrund mehrfach durchgeführter Varianzanalysen wurden die Ergebnisse unter Berücksichtigung einer Alpha-Fehlerkorrektur nach Bonferroni interpretiert. Zur Prüfung der Übereinstimmung der ärztlichen Einschätzung und des Ergebnisses der Testbatterie wurde Cohens Kappa (к) berechnet [29]. Dieser Koeffizient gewichtet das Verhältnis zwischen beobachteter und erwarteter Übereinstimmung zweier Beurteilungen. Bortz und Döring [30] geben einen Minimalwert von $\kappa=0,60$ als „Grenzwert“ an, der für eine gute Beurteiler-Übereinstimmung spricht. Zur Überprüfung der Mittelwertunterschiede bei intervallskalierten Ergebnissen wurde der t-Test genutzt, wobei ein Signifikanzniveau von $p<0,05$ als statistisch signifikant interpretiert wurde. Zur jeweiligen Mittelwertdifferenz wird ergänzend das $95 \%$-Konfidenzintervall berichtet. Als parameterunabhängiger Indikator der Mittelwertunterschiede wurde die Effektstärke (d) [31] unter Verwendung gepoolter Standardabweichungen für unterschiedliche Gruppengrößen berechnet. Alle Analysen wurden mit der Statistiksoftware SPSS 23.0 durchgeführt. 
- Tab. 4 Diagnoseabhängige Ergebnisse in den einzelnen Tests der Testbatterie ( $n=323$ Probanden in der Erprobungsphase).

\begin{tabular}{|c|c|c|c|c|c|}
\hline \multirow{2}{*}{ Test } & \multirow{2}{*}{ Gesamt } & \multicolumn{3}{|c|}{ Diagnosegruppe } & \multirow{2}{*}{$\begin{array}{l}\text { Post-Hoc-Tests } \\
\text { nach Scheffé }\end{array}$} \\
\hline & & RS & cP & SpA & \\
\hline 6-Minuten-Gehtest (Meter), M (SD) & $543(77)$ & $540(79)$ & $546(77)$ & $560(68)$ & n.s. \\
\hline Kniebeugen (Anzahl), M (SD) & $48(24)$ & $47(23)$ & $46(24)$ & $57(23)$ & n.s. \\
\hline Rumpfbeugen (Anzahl), M (SD) & $36(21)$ & $38(21)$ & $33(17)$ & $38(26)$ & n.s. \\
\hline Biering-Sørensen-Test (Sekunden), M (SD) & $75(50)$ & $71(44)$ & $82(57)$ & $86(62)$ & n.s. \\
\hline
\end{tabular}

M... Mittelwert; SD... Standardabweichung; RS = chronischer Rückenschmerz; $\mathrm{CP}=$ chronische Polyarthritiden; SpA=Spondyloarthritiden; $\mathrm{n} . \mathrm{s} .=$ nicht signifikant

> Tab. 5 Leistungsspanne in den Einzeltests ( $n=163$ Probanden der Erprobungsphase).

\begin{tabular}{|c|c|c|c|c|c|c|c|c|c|}
\hline \multirow{2}{*}{ Test } & \multicolumn{9}{|c|}{ Perzentile } \\
\hline & $5 \%$ & $10 \%$ & $25 \%$ & $50 \%$ & $75 \%$ & $90 \%$ & $95 \%$ & Min & Max \\
\hline 6-Minuten-Gehtest (Meter) & 400 & 483 & 517 & 557 & 600 & 632 & 671 & 300 & 694 \\
\hline Kniebeugen (Anzahl) & 12 & 20 & 32 & 50 & 75 & 76 & 79 & 0 & 89 \\
\hline Rumpfbeugen (Anzahl) & 9 & 16 & 24 & 31 & 50 & 75 & 76 & 4 & 96 \\
\hline Biering-Sørensen-Test (Sekunden) & 7 & 14 & 40 & 71 & 104 & 134 & 180 & 0 & 240 \\
\hline Subjektive Belastbarkeit (0-10) & 3 & 3 & 4 & 5 & 6 & 7 & 7 & 1 & 10 \\
\hline
\end{tabular}

Bedeutung der Perzentile: $\mathrm{X}$ \% der Stichprobe haben einen Wert von $\mathrm{Y}$ oder geringerer erreicht; Min = Minimum; Max= Maximum

\section{Ergebnisse}

\section{Stichprobencharakteristik in der Erprobungsphase und Leistungsspanne in den Einzeltests}

Die Betrachtung einzelner Testergebnisse von den 323 Probanden (Alter: $M=49,3$ [ $\pm 8,2$ ] Jahre; $45 \%$ Frauen; Diagnose: 65,3\% RS; $23,1 \%$ cP; $11,6 \% \mathrm{SpA}$ ), die im Rahmen der Interventionsphase die Testbatterie absolvierten, bestätigt eine große Leistungsheterogenität im untersuchten Kollektiv, wobei keine signifikanten diagnosebezogene Leistungsunterschiede zu beobachten waren ( $\triangleright$ Tab. 4). Für die 4 Tests wurden geringe bis mittlere Korrelationen untereinander beobachtet $(r=0,28$ bis $r=0,43 ; p<0,01)$.

\section{Gegenüberstellung von ärztlicher und testbasierter Belastungseinschätzung}

Für den Vergleich der ärztlichen und testbasierten Belastungseinschätzung lagen insgesamt 163 Datensätze vor, bei denen die ärztliche Belastungseinschätzung und die relevanten Fragebogenitems vollständig dokumentiert wurden. Diese Analysestichprobe bestand zur Hälfte aus Frauen (49\%) mit einem durchschnittlichen Alter von 48,9 $( \pm 8,6)$ Jahren und einer ähnlichen Verteilung der Hauptdiagnosen wie in der Entwicklungsphase (59,5\% RS, 25,8\% cP und 14,7\% SpA). Auch in dieser Substichprobe lässt sich die Leistungsvarianz anhand der einzelnen Tests der Testbatterie verdeut- lichen (siehe $>$ Tab. 5). Bei wiederholten Varianzanalysen mit der kleineren Substichprobe ergaben sich wiederum keine signifikanten Unterschiede hinsichtlich der diagnosebezogenen Leistungsvarianz. Für die vier Tests wurden ebenfalls geringe bis mittlere Korrelationen untereinander beobachtet $(r=0,21$ bis $r=0,41 ; p<0,01)$. Von den Probanden wiesen 13,5\% Auffälligkeiten im Gangbild auf, bei 12,3\% wurden Probleme bei der Testdurchführung dokumentiert. Die subjektive Leistungsfähigkeit der Rehabilitanden lag mit $5,2( \pm 1,4)$ im mittleren Bereich.

Von den 163 Rehabilitanden wurden auf Basis der ärztlichen Begutachtung 83 Rehabilitanden als moderat belastbar und 80 Rehabilitanden als intensiv belastbar eingestuft ( $\triangleright$ Tab. 6). Die Testleiter stuften aufgrund der Testbatterie 65 Rehabilitanden als moderat und 98 Rehabilitanden als intensiv belastbar ein. Insgesamt wurden 101 von 163 Rehabilitanden durch beide Einschätzungsverfahren der gleichen Leistungsgruppe ( $n=43$ moderat, $n=58$ intensiv) zugeordnet. Bei mehr als jedem dritten Fall gab es eine gegensätzliche Einschätzung. Cohens Kappa als Maß der Übereinstimmung beider Verfahren betrug $\mathrm{K}=0,24$ (95\% KI: $[0,10 ; 0,40])$.

Hinsichtlich Alter, Geschlecht und Hauptdiagnose zeigten sich zwischen ärztlicher und testbezogener Eingruppierung vergleichbare Charakteristiken ( $\triangleright$ Tab. 7). Bei beiden Varianten waren die als moderat klassifizierten Rehabilitanden durchschnittlich etwas älter; die Diagnosen waren etwa gleich verteilt. Dagegen fanden sich bei den Werten des FFB-Mot größere Mittelwertdifferenzen 
D Tab. 6 Ärztliche und testbasierte Eingruppierung der 163 Probanden nach moderater und intensiver Belastbarkeit (Erprobungsphase).

\begin{tabular}{|l|l|l|l|l|}
\hline & & \multicolumn{2}{|c|}{ Eingruppierung mittels Testbatterie } \\
\hline & & moderat belastbar & intensiv belastbar \\
\hline \multirow{2}{*}{ Ärztliche Eingruppierung } & moderat belastbar & $43(26 \%)$ & $40(25 \%)$ & $\mathbf{8 3}(\mathbf{5 1} \%)$ \\
\hline & intensiv belastbar & $22(14 \%)$ & $58(35 \%)$ & $\mathbf{8 0}(\mathbf{4 9} \%)$ \\
\hline & Gesamt & $\mathbf{6 5}(\mathbf{4 0} \%)$ & $\mathbf{9 8}(\mathbf{6 0} \%)$ & $\mathbf{1 6 3}(\mathbf{1 0 0 \% )}$ \\
\hline
\end{tabular}

In den Zellen ist die jeweilige Anzahl der Rehabilitanden abgebildet; alle dargestellten Anteile (\%) beziehen sich auf die Gesamtstichprobe von 163 Rehabilitanden

und Effektstärken durch die Testallokation im Vergleich zur Allokation durch den Arzt. Deutlich wurde dies an den Effektstärken, die nach ärztlicher Zuteilung im Bereich von 0,23 bis 0,34 lagen und bei der testbezogenen Zuteilung zwischen 0,43 und 0,81 variierten. Vergleichbare Ergebnisse zeigten sich beim IMET und beim SF12, wobei der Unterschied der Mittelwertdifferenzen beim SF-12 am geringsten ausfiel. Für die Ergebnisse der beschwerdebezogenen Merkmale Schmerzen, Erschöpfung und Morgensteifheit fielen die Ergebnisse weniger deutlich aus. Während bei der ärztlichen Zuteilung eine leichte Tendenz zu stärker belasteten Probanden in der moderaten Gruppe auftrat, zeigten sich bei den moderat und intensiv belastbaren Probanden auf Basis der testbezogenen Zuteilung keine Unterschiede hinsichtlich ihrer Schmerzen, Erschöpfung und/oder Steifheit.

Bei Gegenüberstellung der Ergebnisse der 4 motorischen Einzeltests zeigen sich bei der testbasierten Unterteilung deutlich gröBere Mittelwertdifferenzen zwischen den als moderat und intensiv belastbar eingeschätzten Rehabilitanden als im Vergleich zur ärztlichen Unterteilung.

\section{Diskussion}

Das vorgestellte Projekt hatte zum Ziel, eine funktionsbezogene Testbatterie als Entscheidungshilfe für eine leistungsorientierte Gruppenallokation in der rehabilitativen Bewegungstherapie zu entwickeln und zu testen sowie deren potentiellen Mehrwert gegenüber dem Standardverfahren zu ermitteln. Die Herausforderung bestand vor allem in der Erprobung eines ressourcenschonenden Verfahrens nahe an der therapeutischen Praxis, das möglichst einfach für verschiedene Kliniken und Rehabilitandengruppen im Indikationsgebiet der muskuloskeletalen Krankheiten adaptierbar ist.

In der Evaluierungsphase offenbarte sich in den wenigen eingesetzten Tests eine breite Leistungsspanne der untersuchten Rehabilitanden und unterstrich damit die Sinnhaftigkeit einer Leistungsbeurteilung bereits zu Reha-Beginn. Die an der boRN-Studie beteiligten Therapeuten waren von Anfang an in die Testentwicklung involviert und brachten ihre praktischen Erfahrungen u. a. über Vorschläge zum Einsatz bestimmter Testverfahren ein. In der Stichprobe waren sowohl stark in ihrer körperlichen Belastbarkeit eingeschränkte Rehabilitanden als auch Rehabilitanden mit hohem Leistungsniveau vertreten. Dabei waren objektive Merkmale allein, wie z. B. Alter, Geschlecht und Diagnose, wenig aussagekräftig bezüglich der tatsächlichen körperlichen Belastbarkeit der Rehabilitanden.

Während das Arzturteil und die Ergebnisse der Testbatterie vergleichbare moderate und intensive Gruppen hinsichtlich der Alters-, Geschlechts- und Diagnoseverteilung hervorbrachten, zeigten sich deutliche Unterschiede hinsichtlich der Ergebnisse der Einzeltests und der funktionsbezogenen Merkmale im Fragebogen. Die Differenzen der nach den Ergebnissen der Testbatterie gebildeten Gruppen fielen erwartungsgemäß durchweg größer aus als die Differenzen der Gruppen aufgrund des Urteils der Ärzte, die die Testergebnisse nicht kannten. Die geringen Gruppendifferenzen bei der ärztlichen Unterteilung verdeutlichen die leistungsbezogene Vermischung der beiden anvisierten Leistungsgruppen. Dies könnte aber auch dafür sprechen, dass sich auf Basis der ärztlichen Anamnese und Aufnahmeuntersuchung allein die körperliche Belastbarkeit der Rehabilitanden nicht optimal bewerten lässt. Es ist denkbar, dass bei fehlenden objektiven Befunden und Kriterien zur körperlichen Belastbarkeit eine eher defizitorientierte Darstellung der Rehabilitanden die ärztliche Einschätzung in der Aufnahmesituation beeinflussen könnte, auch wenn die Begutachtung zum (erwerbsbezogenen) Leistungsvermögen erst bei Entlassung aus der medizinischen Rehabilitation ansteht. Bei der Durchführung der Testbatterie kann zudem ein gewisser Wettbewerbsfaktor eine Rolle gespielt haben, wodurch sich die Rehabilitanden im direkten Peervergleich ggf. eher auf ihre Stärken als auf ihre Schwächen (und Beschwerden) konzentrierten. Dennoch zeigten sich gerade in den Subskalen des FFB-Mot nach testbezogener Zuteilung größere Mittelwertdifferenzen als bei der ärztlichen Zuteilung. Da es sich beim FFB-Mot um einen Selbsteinschätzungsbogen zu verschiedenen körperlichen Aktivitäten und alltagsbezogenen Funktionen handelt, spiegelt sein Wert die funktionelle Selbsteinschätzung der Patienten wider. Das Ergebnis könnte daher auch dahingehend interpretiert werden, dass die verschiedenen Facetten der körperlichen Belastbarkeit im Rahmen der ärztlichen Anamnese und Untersuchung - möglicherweise aus Zeitgründen - nicht so detailliert thematisiert werden konnten, wie sie im Fragebogen erhoben wurden.

Das gewählte Vorgehen zur Entwicklung der Testbatterie hat sich als geeignet und machbar erwiesen. Es empfiehlt sich vor allem für die klinikspezifische Entwicklung von Bewertungshilfen der körperlichen Belastbarkeit der Rehabilitanden. Eine Cut-Off-Bestimmung für ein Testverfahren anhand der klinikeigenen Klientel kann die Bildung ausgewogener Leistungsgruppen in den bewegungs- 


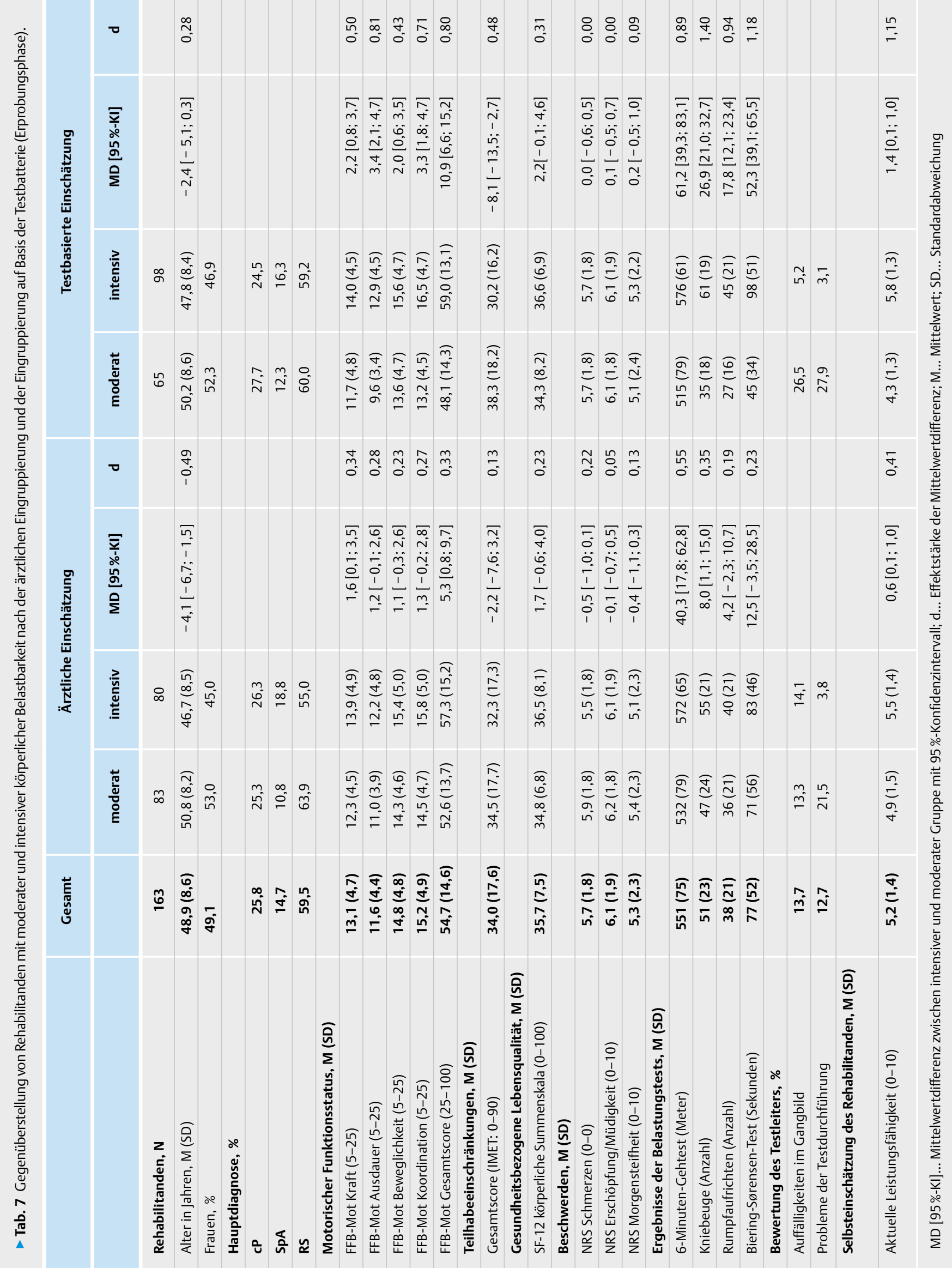


therapeutischen Angeboten begünstigen und somit eine ökonomische Therapieplanung ermöglichen. Darin ist der Vorteil gegenüber der Verwendung testspezifischer Normwerte zu sehen. Der Vorteil der Verwendung solcher Normwerte liegt dagegen in der Vergleichbarkeit von Rehabilitanden auch über die eigenen Klinikgrenzen hinweg, z. B. in einem größeren Klinikverbund oder für einen bestimmten Kostenträger. Aber auch ohne Verwendung eines Cut-Off-Wertes für die Gruppenallokation kann eine Testbatterie wichtige Informationen für Therapieentscheidungen liefern.

Zur Diskussion steht die klinische Relevanz einer leistungsorientierten Ausgestaltung der Sport- und Bewegungstherapie in der medizinischen Rehabilitation. Trainingswissenschaftlich betrachtet sind innerhalb eines 3-wöchigen Rehabilitationsaufenthaltes keine ausgeprägten Leistungssprünge zu erwarten, die allein auf konditionellen Adaptationsprozessen beruhen. Ebenfalls ist die konditionelle Funktionsverbesserung in der Sport- und Bewegungstherapie neben dem Aufbau von Bewegungskompetenz, der Förderung von Bewältigungsprozessen und einer Anbahnung eines aktiven Lebensstils nur eines von mehreren Teilzielen. Aber gerade der knappe Zeitrahmen der medizinischen Rehabilitation und der hohe Umfang bewegungstherapeutischer Angebote im Vergleich zu anderen Therapieformen spricht für eine frühzeitige, ökonomische Diagnostik und eine patientenorientierte Zuweisungssteuerung bewegungstherapeutischer Angebote, um in der vorhandenen Zeitspanne ein optimales Therapieergebnis zu erreichen.

Die Ergebnisse der Evaluierung verdeutlichen, dass sich durch die Testbatterie im Vergleich zur ärztlichen Anamnese die Aufteilung von Rehabilitanden in 2 Leistungsgruppen verbessern ließ. Darüber hinaus könnte eine Wiederholung der Testbatterie am Rehabilitationsende im Rahmen der Qualitätssicherung zur objektivierten Messung des Rehabilitationserfolges auf funktionaler Ebene eingesetzt werden. Nicht zu unterschätzen für den positiven Anschub einer Lebensstiländerung (z. B. hin zu mehr körperlicher Aktivität) sind die motivationalen Aspekte einer objektiven Leistungsmessung. Wer feststellt, dass er durch sein Handeln etwas bewirken kann, entwickelt eine höhere handlungs- und ergebnisbezogene Selbstwirksamkeit und damit eine wichtige motivationale Voraussetzung zu fortgesetzter eigenständiger Sport- und Bewegungsaktivität [32, 33].

Der Mehrwert einer krankheitsübergreifenden, leistungsorientierten Gruppentestung im Rahmen der medizinischen Rehabilitation liegt somit

- in der einfachen objektiven Messung der körperlichen Belastbarkeit zu klinikinternen und/oder externen Vergleichszwecken im Rahmen der Qualitätssicherung,

- in der Optimierung der Zuweisung von Rehabilitanden mit verschiedenen Hauptdiagnosen zu bewegungstherapeutischen Angeboten mit einheitlichem Leistungsanspruch,

- in der Motivierung der Rehabilitanden durch die Rückmeldung messbarer Erfolge und

- in einer stärker funktionsorientierten Ausrichtung der medizinischen Rehabilitation gemäß ICF [34, 35].

\section{Limitationen}

Die Entwicklung und Erprobung der hier beschriebenen Testbatterie erfolgte an Rehabilitanden mit entzündlichen und nicht-entzündlichen muskuloskeletalen Erkrankungen im Heilverfahren unter Trägerschaft der Deutschen Rentenversicherung. Damit waren Rehabilitanden in der Anschlussrehabilitation oder im Rentenalter per se von der Studie ausgeschlossen. Inwiefern eine bzgl. der Hauptdiagnose krankheitsübergreifende Testbatterie auch auf ältere und damit häufiger auch multimorbide Patienten anwendbar wäre und welche Limitierungen und Chancen ein solches Verfahren hätte, muss die zukünftige Forschung beantworten.

Weiterhin liegt der Fokus der Testbatterie auf kraftausdauerorientierten Tests, da im Rahmen der boRN-Studie eine Intervention mit Schwerpunkt im Kraftausdauertraining durchgeführt wurde. Zwar fließt mit dem 6-Minuten-Gehtest auch eine Ausdauerkomponente in die Gesamtbewertung ein, erfährt aber gemäß dem Auswertungsschema nur eine geringe Gewichtung. Die Allokationsfunktion für sporttherapeutische Interventionen mit einem anderen inhaltlichen Schwerpunkt ist somit nicht unmittelbar gegeben.

Die Aussagekraft von Cut-Off-Werten eines Funktionstests für die Gruppenzuteilung ist eng an klinikspezifische Rehabilitandenkollektive gebunden, welche im Idealfall über die Zeit hinweg eine vergleichbare Spanne der körperlichen Leistungsfähigkeit aufweisen. Bei starken Leistungsschwankungen, die sich möglicherweise durch eine sehr heterogene Klinikbelegung ergeben, sind Cut-OffWerte unter Umständen weniger hilfreich, da die Rehabilitanden dann z. B. überwiegend einer Leistungsgruppe zugeteilt werden. Bei einer dichotomen Beurteilung der Leistungsfähigkeit muss zudem berücksichtigt werden, dass Leistungsunterschiede sich durch die Testung zwar reduzieren lassen, aber auch innerhalb der moderaten und intensiven Gruppen eine gewisse Leistungsheterogenität zu erwarten ist. Eine solche Testbatterie sollte daher als Entscheidungshilfe mit zusätzlichem Informationsgewinn zur Optimierung des therapeutischen Prozesses verstanden und eingesetzt werden. Der praxisbezogene Mehrwert deutet sich durch die hier berichtete Evaluierung zwar an, es fehlt jedoch an einem kontrollierten Studiendesign zur Überprüfung dieser Annahme, das neben den Subtests der Testbatterie und der Selbsteinschätzung der Rehabilitanden auch weitere objektive Verfahren der Leistungsbestimmung berücksichtigt (z. B. Spiroergometrie, BelastungsEKG, apparative isokinetische Kraftmessung), da sonst die Gefahr eines Ringschlusses besteht.

Vor der Entwicklung eines diagnostischen Verfahrens zur Abschätzung der körperlichen Belastbarkeit der Probanden und dessen flächendeckendem Einsatz sollten Testaufwand und tatsächlicher Mehrwert einer solchen Testung daher abgewogen werden. Auch wenn der Einsatz objektiver diagnostischer Maße im Rahmen der Sport- und Bewegungstherapie grundsätzlich zu fördern und zu befürworten ist [3], hängt der Nutzen dieses Vorgehens auch von Strukturparametern, wie z. B. von der Klinikgröße und dem Patientenaufkommen, sowie von den organisatorischen Möglichkeiten der Durchführung leistungsgestufter Trainingsgruppen ab.

\section{Schlussfolgerung}

Zusammenfassend liefert die Studie praxisrelevante Erkenntnisse für die sport- und bewegungstherapeutische Weiterentwicklung. So zeigte sich im gesamten Entwicklungsprozess bei den untersuchten Rehabilitanden in den eingesetzten Testverfahren eine breite Leistungsspanne bzgl. Kraft und Ausdauer. Alter, Geschlecht oder Diagnose allein erwiesen sich dabei als wenig aussagekräfti- 
ge Indizien für die Beurteilung der körperlichen Belastbarkeit. Bei der Einschätzung zur Belastbarkeit der Rehabilitanden in der Erprobungsphase deckten sich das Arzturteil und die Ergebnisse der Testbatterie nur teilweise. Die verschiedenen Gruppen mit moderater bzw. intensiver Belastbarkeit zeigten unterschiedlich große Differenzen hinsichtlich der geprüften funktionsbezogenen Parameter. Die erprobte Testbatterie konnte die Unterteilung in moderat und intensiv belastbare Rehabilitanden gegenüber dem Standardverfahren (Arzturteil) auf Grundlage der generierten Daten scheinbar verbessern. Somit ist die Unterteilung der Rehabilitanden in 2 Trainingsgruppen mit unterschiedlichem Leistungsanspruch hier offenbar besser gelungen als durch die Unterteilung basierend auf den Informationen, die sich im Rahmen der ärztlichen Aufnahmeuntersuchung generieren lassen. Insofern könnte eine leistungsorientierte Testung einen Mehrwert zur Einschätzung der funktionsbezogenen Belastbarkeit der Rehabilitanden bieten und als ergänzendes Instrument für sport- und bewegungstherapeutische Therapieentscheidungen hilfreich sein.

Insgesamt hat sich das beschriebene Vorgehen für die klinikspezifische Entwicklung einer Entscheidungshilfe der körperlichen Belastbarkeit der Rehabilitanden bei einem im Zeitverlauf ähnlichen Rehabilitandenkollektiv innerhalb der Klinik als geeignet erwiesen. Die Orientierung an klinikinternen vs. (extern) normierten Testwerten hängt vom jeweiligen Einsatzziel ab. Zwar sollte der generelle Aufwand der Entwicklung und Implementierung einer objektivierenden Diagnostik für den Bereich der Sport- und Bewegungstherapie nicht unterschätzt werden. Durch den hohen Anteil dieser Therapien an der Gesamttherapiezeit und deren damit verbundenen Stellenwert scheint der Aufwand jedoch gerechtfertigt.

\section{Danksagung}

Unser Dank gilt allen Rehabilitandinnen und Rehabilitanden, die durch ihre Teilnahme und Mitarbeit die Studie ermöglicht haben. Besonders bedanken wir uns bei den therapeutischen Teams des Rehazentrums Bad Eilsen und der CELENUS Teufelsbad Fachklinik Blankenburg für die Unterstützung und das unermüdliche Engagement bei der Studiendurchführung.

Interessenkonflikt

Die boRN-Studie wurde durch die Deutsche Rentenversicherung Bund gefördert.

\section{Literatur}

[1] Brüggemann S, Sewöster D. Bewegungstherapeutische Versorgung in der medizinischen Rehabilitation der Rentenversicherung. B \& G 2010; 26: 266-269

[2] Brüggemann S, Sewöster D. Bewegungstherapeutische Versorgung in der medizinischen Rehabilitation der Rentenversicherung - Vergleich der Jahre 2007 und 2012. In: Deutsche Rentenversicherung Bund., (Hrsg.). 24. Rehabilitationswissenschaftliches Kolloquium. Psychische Störungen - Herausforderungen für Prävention und Rehabilitation; 2015: 231-234
[3] Arbeitsgruppe „Bewegungstherapie“. Ziele und Aufgaben der Arbeitsgruppe „Bewegungstherapie“ in der Deutschen Gesellschaft für Rehabilitationswissenschaften (DGRW). Rehabilitation 2009; 48: 252-255

[4] Pfeifer K, Sudeck G, Brüggemann S et al. DGRW-Update. Bewegungstherapie in der medizinischen Rehabilitation - Wirkungen, Qualität, Perspektiven. Rehabilitation 2010; 49: 224-236

[5] Sudeck G, Pfeifer K. Bewegung in der Rehabilitation - ICF-Bezug, Kompetenzorientierung, Nachhaltigkeit. Public Health Forum 2013; 21

[6] Geidl W, Hofmann J, Gohner W et al. Verhaltensbezogene Bewegungstherapie - Bindung an einen körperlich aktiven Lebensstil. Die Rehabilitation 2012; 51: 259-268

[7] Arbeitsgruppe „Bewegungstherapie“. Empfehlungen der Deutschen Gesellschaft für Rehabilitationswissenschaften (DGRW) zur Weiterentwicklung der „Rahmenvereinbarung über den Rehabilitationssport und das Funktionstraining" der Bundesarbeitsgemeinschaft für Rehabilitation (BAR) vom 1.1.2011. Die Rehabilitation 2016; 55: 130-132

[8] Semrau J, Hentschke C, Buchmann J et al. Long-term effects of interprofessional biopsychosocial rehabilitation for adults with chronic non-specific low back pain: A multicentre, quasi-experimental study. PloS one 2015; 10: e0118609

[9] Geidl W, Semrau J, Pfeifer K. Health behaviour change theories: contributions to an ICF-based behavioural exercise therapy for individuals with chronic diseases. Disability and rehabilitation 2014; 36: 2091-2100

[10] Deck R, Schramm S, Huppe A. Begleitete Eigeninitiative nach der Reha („neues Credo“) - ein Erfolgsmodell? Die Rehabilitation 2012; 51: 316-325

[11] Mattukat K, Rennert D, Brandes I et al. Short- and long-term effects of intensive training and motivational programme for continued physical activity in patients with inflammatory rheumatic diseases. European Journal of Physical and Rehabilitation medicine 2014; 50: 395-409

[12] Geidl W, Hendrich S, Semrau J et al. Entwicklung evidenzgesicherter Konzepte für die Bewegungstherapie in der Rehabilitation. Abschlussbericht 2009:

[13] Geidl W, Streber R, Deprins J et al. Bewegungstherapie in der medizinischen Rehabilitation: inhaltliche Ausrichtung und Standardisierungsgrad. Ergebnisse einer bundesweiten Bestandaufnahme. In: Deutsche Rentenversicherung Bund., (Hrsg.). 25. Rehabilitationswissenschaftliches Kolloquium. Gesundheitssystem im Wandel - Perspektiven der Rehabilitation 2016: 316-317

[14] Tittlbach S, Knyrim H, Baumeister ] et al. Motorische Tests. In: Banzer W, Pfeifer K, Vogt L. Hrsg. Funktionsdiagnostik des Bewegungssystems in der Sportmedizin. Berlin, Heidelberg: Springer Berlin Heidelberg; 2004: 71-86

[15] Reibis RK, Schlitt A, Glatz ] et al. Rehabilitation bei Herzinsuffizienz. Die Rehabilitation 2016; 55: 115-129

[16] Streibelt M, Buschmann-Steinhage R. Ein Anforderungsprofil zur Durchführung der medizinisch-beruflich orientierten Rehabilitation aus der Perspektive der gesetzlichen Rentenversicherung. Die Rehabilitation 2011; 50: 160-167

[17] Bös K., (Hrsg.). Handbuch motorische Tests. Sportmotorische Tests, motorische Funktionstests, Fragebogen zur körperlich-sportlichen Aktivität und sportpsychologische Diagnoseverfahren. 2. Aufl. Göttingen: Verl. für Psychologie; 2001

[18] Wydra G. Eine problemorientierte Diagnosestrategie für die Sporttherapie. In: Schüle K, Huber G, (Hrsg.). Grundlagen der Sportund Bewegungstherapie. Köln: Deutscher Ärzteverlag; 2012: 182-194

[19] Mattukat K, Golla A, Mau W. Gestufte bewegungsorientierte Rehabilitation und Nachsorge bei Patienten mit entzündlichen und nicht-entzündlichen Erkrankungen des Bewegungssystems (boRN). Abschlussbericht. Halle (Saale). 2014; Online verfügbar unter: http:// www.forschung-patientenorientierung.de/files/born_abschlussbe richt_18.11.2014_endversion.pdf zuletzt geprüft am 29.08.2016 
[20] Bös K, Pfeifer K, Stoll O et al. Testtheoretische Grundlagen. In: Bös K, (Hrsg.). Handbuch motorische Tests. Sportmotorische Tests, motorische Funktionstests, Fragebogen zur körperlich-sportlichen Aktivität und sportpsychologische Diagnoseverfahren. 2. Aufl. Göttingen: Verl. für Psychologie; 2001: 531-571

[21] Enright PL, Sherrill DL. Reference equations for the six-minute walk in healthy adults. American Journal of Respiratory and Critical Care Medicine 1998; 158: 1384-1387

[22] Demoulin C, Vanderthommen M, Duysens C et al. Spinal muscle evaluation using the Sorensen test: A critical appraisal of the literature. Joint, bone, spine: revue du rhumatisme 2006; 73: 43-50

[23] Kinzey S], Armstrong CW. The reliability of the star-excursion test in assessing dynamic balance. The Journal of Orthopaedic and Sports Physical Therapy 1998; 27: 356-360

[24] Löllgen H. Das Anstrengungsempfinden (RPE, Borg-Skala). Deutsche Zeitschrift für Sportmedizin 55: 299-300

[25] Hanna M, Tilscher H, Weich C. Computerisierte Dokumentation von klinischen und manuellen Befunden. Manuelle Med 1991; 29

[26] Bös K, Abel T, Woll A et al. Der Fragebogen zur Erfassung des motorischen Funktionsstatus (FFB-Mot). Diagnostica 2002; 48 : 101-111

[27] Deck R, Mittag O, Hüppe A et al. Index zur Messung von Einschränkungen der Teilhabe (IMET) - Erste Ergebnisse eines ICF-orientierten Assessmentinstruments. Praxis Klinische Verhaltensmedizin und Rehabilitation 2007; 20: 113-120
[28] Bullinger M, Morfeld M, Kohlmann T et al. Der SF-36 in der rehabilitationswissenschaftlichen Forschung - Ergebnisse aus dem Norddeutschen Verbund für Rehabilitationsforschung (NVRF) im Förderschwerpunkt Rehabilitationswissenschaften. Die Rehabilitation 2003; 42: 218-225

[29] Grouven U, Bender R, Ziegler A et al. Der Kappa-Koeffizient. Deutsche medizinische Wochenschrift 2007; 132 (Suppl 1): 8

[30] Bortz ], Döring N. Forschungsmethoden und Evaluation. Berlin, Heidelberg: Springer Berlin Heidelberg; 2006

[31] Cohen J. Statistical power analysis for the behavioral sciences. 2. Aufl. Hillsdale, N.J.: L. Erlbaum Associates; 1988

[32] Bandura A. Health promotion by social cognitive means. Health education \& behavior: The official publication of the Society for Public Health Education 2004; 31: 143-164

[33] Lippke S, Renneberg B. Theorien und Modelle des Gesundheitsverhaltens. In: Renneberg B, Hammelstein P, (Hrsg.). Gesundheitspsychologie. Heidelberg: Springer Medizin; 2006: 35-60

[34] Farin E. Patientenorientierung und ICF-Bezug als Herausforderungen für die Ergebnismessung in der Rehabilitation. Die Rehabilitation 2008; 47: 67-76

[35] Mau W, Beyer W, Ehlebracht-Konig I et al. Treat to participation. DGRh-Positionspapier zur nachhaltigen Verbesserung des funktionalen Gesundheitszustandes von Rheumakranken. Zeitschrift fur Rheumatologie 2015; 74: 553-557 